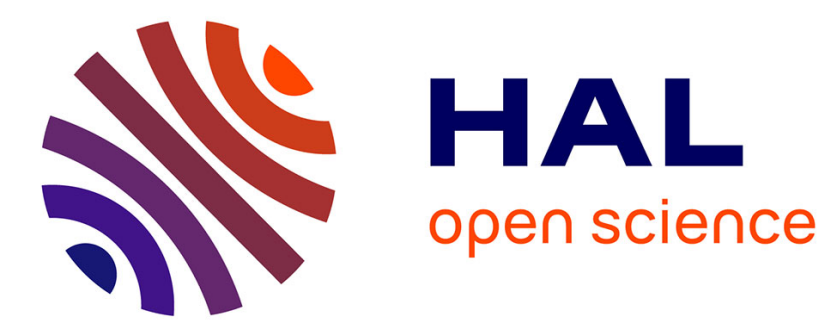

\title{
Non-intrusive coupling: an attempt to merge industrial and research software capabilities
}

\author{
Olivier Allix, Lionel Gendre, Pierre Gosselet, Guillaume Guguin
}

\section{To cite this version:}

Olivier Allix, Lionel Gendre, Pierre Gosselet, Guillaume Guguin. Non-intrusive coupling: an attempt to merge industrial and research software capabilities. Dana Mueller-Hoeppe, Stefan Loehnert and Stefanie Reese. Recent developments and innovative applications in computational mechanics, Springer, pp.125-133, 2011. hal-00591345

\section{HAL Id: hal-00591345 \\ https://hal.science/hal-00591345}

Submitted on 9 May 2011

HAL is a multi-disciplinary open access archive for the deposit and dissemination of scientific research documents, whether they are published or not. The documents may come from teaching and research institutions in France or abroad, or from public or private research centers.
L'archive ouverte pluridisciplinaire HAL, est destinée au dépôt et à la diffusion de documents scientifiques de niveau recherche, publiés ou non, émanant des établissements d'enseignement et de recherche français ou étrangers, des laboratoires publics ou privés. 


\title{
Non-intrusive coupling: an attempt to merge industrial and research software capabilities
}

\author{
Olivier Allix, Lionel Gendre, Pierre Gosselet and Guillaume Guguin
}

\begin{abstract}
I have had the great pleasure to cooperate since several years now closely with Peter Wriggers who has been several times invited in LMT-Cachan. I have always been impressed by the profound understanding and practical knowledge of Peter regarding computational and material mechanics. I know that Peter has a deep concern regarding the application of fundamental researches which is the motivation of this paper. We hope to connect it more closely in the future to the seminal work of Peter, especially regarding contact and multiscale stochastic modeling of heterogeneous materials and damage. The recently accepted International Research Training Group Virtual Material and Structures and their Validation is therefore an exceptional possibility for us to continue and reinforce a close relationship with Peter, a great scientist and a great friend.
\end{abstract}

\begin{abstract}
In computational mechanics, it is often difficult to test research innovations on industrial problems because of software limitations: many of the commercial finite element packages commonly used in the industry lack flexibility and openness, whereas in-house research developments are usually very specific and may lack features required for "real-life" industrial simulations. Non-intrusive coupling is a tentative answer to this problem. It consists in introducing local enhancements and refinements into an existing industrial problem through a separate nonlinear local model that comes with its own solver; the two models are coupled by the means of an iterative exchange algorithm inspired from domain decomposition methods and multiphysics solution techniques, using both models and solvers without any modification. So far, the method has been implemented around the finite element package Abaqus and has been used to introduce local plasticity and geometric details into a linear elastic global problem. While current developments include the simulation of localized damage in slender composite structures, we think that the method could be adapted to a wide class of problems including hybrid experimentalsimulation approaches.
\end{abstract}

\section{Introduction}

In the last decade, many innovative modelling or solution techniques have been introduced in the field of computational mechanics. These techniques, such as enriched finite elements or multiscale models, enable performing complex simulations

Olivier Allix, Lionel Gendre, Pierre Gosselet, Guillaume Guguin

LMT-Cachan, 61 avenue du Président Wilson, F-94235 Cachan Cedex, FRANCE e-mail: allix,gendre,gosselet,guguin@1mt.ens-cachan.fr 
that are out of reach of conventional finite element analysis (FEA) tools, in terms of computational or human costs. However, although these techniques have proved their performance by extensive testing on academic applications, they are scarcely applied on actual industrial problems because they cannot be conveniently implemented into commercial FEA software packages, which are the basis of most industrial computational environments.

Non-intrusive coupling [11, 12] is a tentative answer to these limitations. It takes advantage of the fact that, in many industrial simulations, sources of difficulties (which are usually nonlinear phenomena, sometimes occurring at fine scales) are localized in small regions, and that the innovative techniques mentioned above were specifically designed to overcome such difficulties efficiently. Thus, the essential idea of non-intrusive coupling is to enhance an existing industrial simulation, that involves a complex model data set and a commercial FEA solver, by the means of a separate local model that is analyzed with its own dedicated solver. This way, the local model may contain innovative features that cannot be implemented conveniently into the commercial solver. The term "non-intrusive" means that in the process, neither the models nor the solvers need to be modified; they are used as "black boxes", and a script is used to run the analyses and exchange displacements and forces between them.

Of course, this non-intrusive framework also has a significant drawback: one has to do with the limitations of the commercial FEA solver that is used. This can impact performance, particularly when using software that are not optimized for implicit solver coupling schemes. However, we believe that this possibly non-optimal performance is a fair price to pay for the convenience of such a black-box tool; in addition, depending on the solver, several adjustements can be performed to reduce computational costs dramatically. At the moment, the computational efficiency of such a non-intrusive strategy is still an open question. What is certain is that this framework provides a way of performing enriched simulations for which no "monolithic" software is available at the present time.

The rest of this chapter is organized as follows. Section 2 reviews the essential ideas of non-intrusive coupling and the different ways it can be made more efficient. Section 3 presents an application of this strategy to localized plasticity and first results on damage problems.

\section{The general principles of non-intrusive coupling}

The proposed analysis strategy starts from an existing "industrial" model, analyzed using commercial FEA software; its behaviour is supposed to be completely linear (linear elasticity under small perturbations without contacts) and static. This model is called the global model and is schematized on Figure 1(a).

Let us assume that in reality, nonlinear phenomena may occur in a small region of the structure $\Omega$, denoted $\Omega_{I}$ and called the area of interest; in the remaining region $\Omega_{C}=\Omega \backslash \Omega_{I}$, called the complement area, the global model is assumed to 
be valid. In order to take those nonlinear phenomena into account, we would like to use innovative techniques or models that cannot be implemented conveniently into the global solver, as explained in the introduction. In addition, we suppose those phenomena may interact with small local details (such as holes or cracks) that were omitted in the global model.

Therefore, we suggest defining a separate local model, limited to $\Omega_{I}$ alone, that contains all the desired enhancements and is analyzed using its own dedicated nonlinear solver.This model is schematized on Figure 1(b). In this article, it is supposed to possess a standard finite element formulation and to be geometrically and kinematically compatible with the global model on the interface $\Gamma=\Omega_{I} \cap \Omega_{C}$. However, this is a simplification rather than a fundamental limitation of the method, and noncompatible discretizations could very well be used as long as appropriate transfer operators are defined (for example, using mortar techniques [4]).

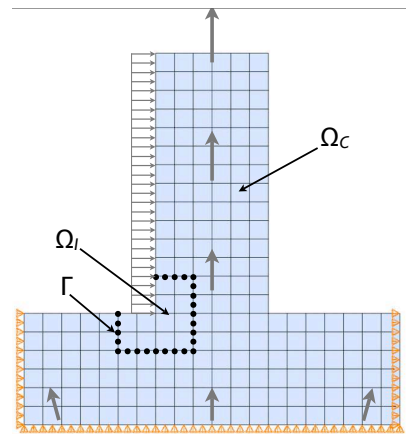

(a) Global

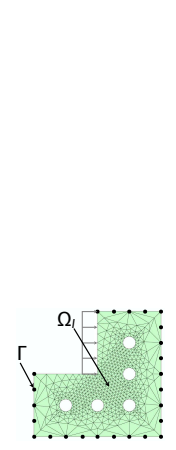

(b) Local

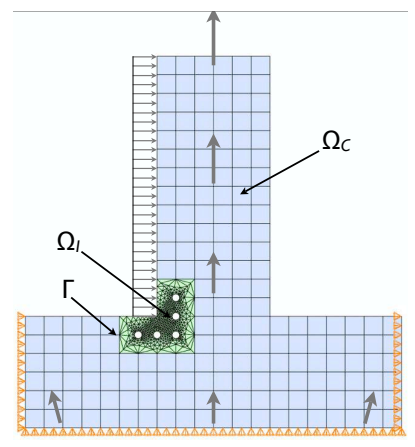

(c) Reference

Fig. 1 Global, local and reference problems

Starting from these models, a coupling method is then used. The following sections present the two main ideas of this method.

\subsection{Piecewise substitution}

Though the two models are overlapping, contrary to classical "patch" methods $[2,3,8,10]$ which define the solution as a combination of a global and a local term, we prefer to refer to non-overlapping formulations (as used in fluid-structure interaction [22], multiscale simulations [25] and nonlinear domain decomposition methods $[7,21]$ ) which limit the exchanges to surface data and seem more prone to the separate handling of nonlinearity. Therefore, we wish to eliminate the overlap

from the formulation. For that purpose, the reference problem that we wish to solve is defined by piecewise substitution of the local model into the global model, as shown on Figure 1(c). Likewise, the solution to this problem is sought by piecewise 
substitution of the local solution into the global solution, that is:

$$
s_{\left.\right|_{\Omega_{I}}}=s^{L} \quad \text { and } \quad s_{\left.\right|_{\Omega_{C}}}=s_{\left.\right|_{\Omega_{C}} ^{G}}^{G}
$$

where $s$ is the set of the solution fields (ie. the displacement field and the Cauchy stress field) and superscripts $G$ and $L$ respectively denote quantities from the global and the local problems. In other terms, the global solution that lies "under" the local model is not retained: the overlap is eliminated.

This way, the solution to the reference problem can be obtained from the two models and solvers by finding two solutions $s^{L}$ and $s^{G}$ such that (see left column):

\begin{tabular}{|l|l|l|}
\hline 1. & $\begin{array}{l}s^{L} \text { verifies every equation written in } \Omega_{I} \\
\text { and its boundary. }\end{array}$ & $h^{L}\left(w^{L}\right)=\lambda^{L}$ \\
\hline 2. & $\begin{array}{l}\text { the restriction of } s^{G} \text { to } \Omega_{C} \text { verifies every } \\
\text { equation written in } \Omega_{C} \text { and its boundary }\end{array}$ & $S_{C}^{G} w^{G}-b_{C}^{G}=\lambda_{C}^{G}$ \\
\hline 3. & $s^{L}$ and $s^{G}$ match on $\Gamma$ & $\begin{array}{l}w^{G}=w^{L} \\
\lambda_{C}^{G}+\lambda^{L}=0\end{array}$ \\
\hline
\end{tabular}

The right column corresponds to the condensed version of the equation, where $w$ is the interface nodal displacement and $\lambda$ the interface nodal reaction force. The linear operator $S_{C}^{G}$ is the Schur complement of the complement area (from the global model) and $b_{C}^{G}$ is the associated condensed right-hand side vector. The nonlinear operator $h^{L}$ formally represents the local problem's reaction to prescribed interface displacements.

This set of three conditions is called the global-local formulation and its solution is trivially equal to the reference solution (assuming it is unique). It defines a surface coupling between the two models. The corresponding equations can be found in [11] in a continuous form, and in [12] in a discrete, condensed form as above. From a computational point of view, a crucial advantage of this formulation is the ability to use a nonlinear local solver to handle local nonlinearity, instead of relying on global iterations only; this principle, as used in $[7,21]$ in presence of localized but pronounced nonlinearity, can lead to huge savings in computational costs.

\subsection{Iterative coupling}

To enforce this coupling and solve the global/local formulation, several approaches can be imagined. In order to be both exact and non-intrusive at a reasonable cost, using an iterative algorithm is the most relevant choice, as suggested in early literature on global/local analysis $[18,23]$. In accordance to the non-intrusive framework, the algorithm only consists in computing the responses to prescribed loads on the different models; no direct matrix manipulations are used. To meet those requirements, we have chosen a simple modified Newton method on interface quantities. This method starts from the initial elastic solution, then each iteration goes as follows. 
1. Local analysis: the local nonlinear problem is solved, with part of the current global solution prescribed as a boundary condition on $\Gamma$. This condition can be prescribed displacements, prescribed nodal forces or a mixed condition, as it will be detailed below. For example, when using the displacement condition, we compute the reaction $\lambda^{L}$ as:

$$
\lambda^{L}=h^{L}\left(w^{L}\right)
$$

2. Residual computation: an interface load vector called the residual is computed. It measures the non-verification of interface coupling equations. Convergence is tested here: if the residual's norm is small enough, iterations are stopped. For example, if prescribed displacements were used, then the residual is the sum of nodal reaction forces between the two models (which should be zero if they were balanced):

$$
r=\lambda^{L}+\lambda_{C}^{G}
$$

where $\lambda_{C}^{G}$ is extracted from the current global solution, either by classical postprocessing using elementary integration of stresses or by using an additional linear model to get reaction forces to the current global displacements.

3. Global correction: otherwise, the residual is injected into the global problem as an additional interface load. This is done by first solving a corrective global problem loaded only with the residual (all other loads and boundary conditions are set to zero) :

$$
\Delta w^{G}=\left(S_{C}^{G}+S_{I}^{G}\right)^{-1} r
$$

This computation is analogous to the linear step of a modified Newton iteration. Therefore, the effectiveness of the correction step can be improved at no extra cost by using a quasi-Newton update formula such as SR1 or BFGS [1, 19], written in a non-intrusive form as shown in [11]. Finally, the corresponding corrective solution $\Delta s^{G}$ is added to the current global solution before going back to step 1 .

This class of algorithms has two important properties. First, if the algorithm converges, it is easy to prove that its limit is the fully coupled solution - that is, the reference solution $[11,12]$. Therefore, the coupling method is reliable: the error can be estimated through the norm of the residuals and reduced as much as needed. Second, the method is indeed non-intrusive since the solvers operate as black boxes, and the model data sets are never modified. It only requires sending boundary conditions to the local solver or additional loads to the global solver, and reading interface nodal displacements and forces which are routine FEA operations. As a consequence, the algorithm's implementation should be light and can use a high-level scripting language as provided by many FEA packages.

\subsection{Choice of the interface boundary condition for the local step}

The simplest boundary condition that can be used on the local model is prescribed displacements, the value of which is extracted from the current global solution. This 
choice enables to work with kinematically admissible displacement fields. For that reason, it is popular in global/local $[6,18,23]$ or multiscale analysis $[9,20]$, and submodelling with displacement conditions is natively available in many commercial FEA packages. Algorithms using this condition were studied in [11] for local plasticity.

However, prescribing a displacement field is arguably not the most realistic way of emulating the influence of the rest of the structure on the local model. Robin conditions are known to be more efficient. They have been experimented in the field of global/local analysis [13,14] and widely studied in domain decomposition methods $[15,17]$. They consist in prescribing a linear combination of displacement and efforts; the factor that appears in this linear combination is an interface stiffness matrix. The choice of this matrix has a huge impact on performance. Intuitively, it emulates the "mechanical impedance" of the rest of the structure, and it is wellknown that it should give a correct approximation of the Schur complement of the linear region's contribution. In [12] we proposed a two-scale approximation where the macro part is evaluated by taking the global model's response to selected loads that represent "long-range" effects, whereas the micro part is given by the Schur complement of a narrow strip of elements adjacent to the interface.

\section{Examples using Abaqus/Standard}

We consider the 2D toy problem represented on Figures 1(a) and 1(b). The local problem's constitutive model is elastic-plastic with linear isotropic hardening; the load is applied in one single increment because of software constraints, and its intensity is such that the elastic limit is slightly exceeded in the area of interest.

To assess the coupling scheme's performance, we solved the reference problem and computed, at each iteration, the relative error on the maximum cumulative plastic strain (with respect to its reference value). This particular quantity was chosen because it is a common goal of the analysis on many elastic-plastic applications (such as estimating the lifespan of ductile structures submitted to cyclic loadings) and because it is highly sensitive to the local model's boundary condition.

The evolution of this relative error during the iterations is shown on Figure 2, for four different variants of the algorithm (with prescribed displacements or Robin conditions, with or without quasi-Newton acceleration). It appears that except for the simplest version (prescribed displacements without acceleration), all variants converge very quickly: an error of $10^{-3}$ on the maximum plastic strain (which is a quite strict threshold, by engineering standards) is reached after 2 or 3 iterations. The corresponding cumulative plastic strain maps are shown on Figure 3, at three instants of the strategy.

As a first study on damage problems, we also considered the same geometry with an isotropic damage law (with bounded rate of damage). For moderate damage, the method behaves the same way (slow convergence for basic displacement approach, effective acceleration with SR 1 and better performance with mixed conditions). The 


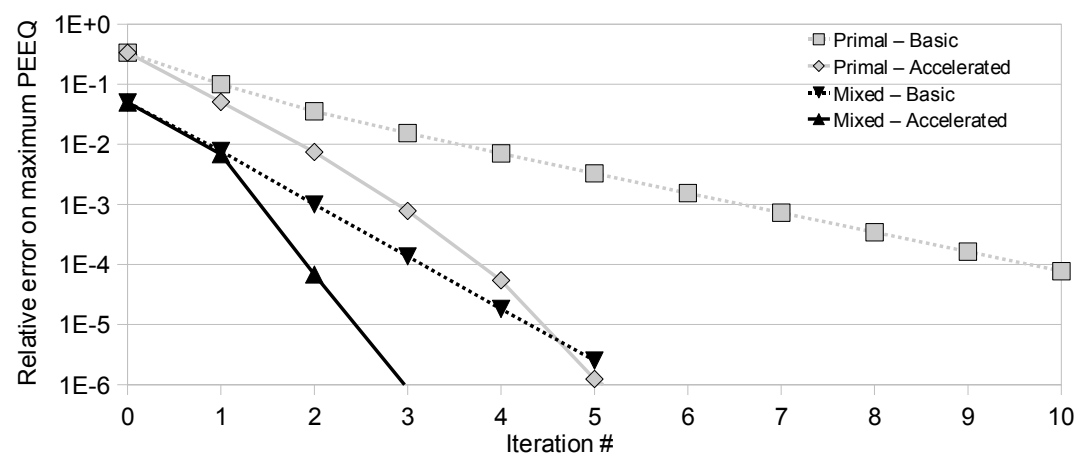

Fig. 2 Convergence of maximum plastic strains on the 2D example with localized plasticity

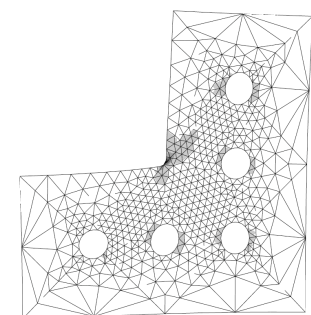

(a) Initial, displacements

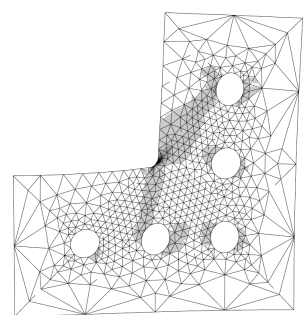

(b) Initial, mixed

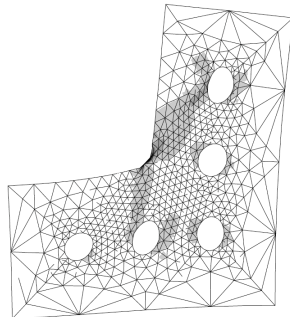

(c) Converged (reference)

Fig. 3 Cumulative plastic strain maps at the first iteration and at convergence

softening associated to damage might be the source of many difficulties that we have to deal with, like localization, instabilities (which require a local control), and dependency on the interface's location.

\section{Conclusion}

We proposed a coupling approach to enhance a global linear model by a local model with refined geometry and non-linear constitutive equation. The approach is nonintrusive so that it can link industrial FEA software to in-house research code. Performance can be improved by using relevant boundary conditions on the local model and using acceleration techniques, leading to an efficient method which was validated on large industrial 3D localized plasticity problems. Prospects concern coupling between plates and 3D models, local treatment of many loading steps, application to damage problems like delamination in composites and to problems involving contact [24] in both local and global models. 


\section{References}

1. Akgün MA, Garcelon JH, Haftka RT (2001) Fast exact linear and nonlinear structural reanalysis and the Sherman-Morrison-Woodbury formulas. Int J Num Meth Engng 50:1587-1606.

2. Babuska I, Andersson B (2005) The splitting method as a tool for multiple damage analysis. SIAM J Sci Comp 26(4):1114-1145

3. Ben Dhia H (1998) Multiscale mechanical problems: the Arlequin method. Comptes-rendus de l'Académie des Sciences IIb(326):899-904

4. Bernardi C, Maday Y, Patera AT (1991) A new nonconforming approach to domain decomposition: the mortar element method. In: Brézis H, Lions JL (eds) Nonlinear partial differential equations and their applications, Collège de France seminar. Pitman, London

5. Boucard PA, Ladevèze P, Poss $M$, Rougée P (1997) A nonincremental approach for large displacement problems. Comp Struct 64(1-4):499-508

6. Cormier NG, Smallwood BS, Sinclair GB, Meda G (1999) Aggressive submodelling of stress concentrations. Int J Num Meth Engng 46:889-909

7. Cresta P, Allix O, Rey C, Guinard S (2007) Nonlinear localization strategies for domain decomposition methods: application to post-buckling analyses. Comp Meth Appl Mech Engng 196(8):1436-1446

8. Düster A, Rank E, Steinl G, Wunderlich W (1999) A combination of an h- and a p-version of the finite element method for elastic-plastic problems. In: Wunderlich W (ed) ECCM '99. CD-ROM proceedings of the European Conference on Computational Mechanics. Munich.

9. Feyel F, Chaboche JL (2000) $\mathrm{FE}^{2}$ multiscale approach for modelling the elastoviscoplastic behavior of long fiber SiC/Ti composite materials. Comp Meth Appl Mech Engng 126:17-38

10. Fish J (1992) The $s$-version of the finite element method. Comp Struct 43(3):539-547

11. Gendre L, Allix O, Gosselet P, Comte F (2009) Non-intrusive and exact global/local techniques for structural problems with local plasticity. Comp Mech 44:233-245

12. Gendre L, Allix O, Gosselet P (2010) A two-scale approximation of the Schur complement and its use for non-intrusive coupling. Submitted to: Int J Num Meth Engng.

13. Hirai I, Wang BP, Pilkey WD (1984) An efficient zooming method for finite element analysis. Int J Num Meth Engng 20:1671-1683

14. Jara-Almonte CC, Knight CE (1988) The specified boundary stiffness and force (SBSF) method for finite element subregion analysis. Int J Num Meth Engng 26:1567-1578

15. Ladevèze P, Dureisseix D (2000) A micro/macro approach for parallel computing of heterogeneous structures. Int J Comp Civil Struct Engng 1:18-28

16. Ladevèze P, Simmonds $\mathrm{J}$ (1998) New concepts for linear beam theory with arbitrary geometry and loading. Eur J Mech - A/Solids 17(3):377-402

17. Magoulès F, Roux FX, Series L (2006) Algebraic approximations of Dirichlet-to-Neumann maps for the equations of linear elasticity. Comp Meth Appl Mech Engng 195:3742-3759

18. Mao KM, Sun CT (1991) A refined global-local finite element analysis method. Int J Num Meth Engng 32:29-43

19. Matthies H, Strang G (1979) The solution of nonlinear finite element equation. Int J Num Meth Engng 14(11):1613-1626

20. Oden JT, Zohdi TI (1997) Analysis and adaptive modeling of highly heterogeneous structures. Comp Meth Appl Mech Engng 148:367-392

21. Pebrel J, Rey C, Gosselet P (2008) A nonlinear dual domain decomposition method: application to structural problems with damage. Int J Multiscale Comp Engng 6:251-262

22. Piperno S, Farhat C, Larrouturou B (1995) Partitioned procedures for the transient solution of coupled aeroelastic problems. Comp Meth Appl Mech Engng 20:1638-1685

23. Whitcomb JD (1991) Iterative global-local finite element analysis. Comp Struct 40(4):10271031

24. Wriggers P (2006) Computational Contact Mechanics. (2nd ed) Springer Verlag: Heidelberg

25. Zohdi TI, Wriggers P, Huet C (2001) A method of substructuring large-scale computationalmicromechanical problems. Comp Meth Appl Mech Eng 190(4344):56395656 